\title{
Communication
}

\section{$30 \mathrm{GHz}$ optoelectronic mixing in CVD graphene}

Alberto Montanaro, Sana Mzali, Jean-Paul Mazellier, Odile Bezencenet, Christian Larat, Stephanie Molin, Loïc Morvan, Pierre Legagneux, Daniel Dolfi, Bruno Dlubak, Pierre Seneor, Marie-Blandine Martin, Stephan Hofmann, John Robertson, Alba Centeno, and Amaia Zurutuza Nano Lett., Just Accepted Manuscript • DOI: 10.1021/acs.nanolett.5b05141 • Publication Date (Web): 04 Apr 2016

Downloaded from http://pubs.acs.org on April 6, 2016

\section{Just Accepted}

"Just Accepted" manuscripts have been peer-reviewed and accepted for publication. They are posted online prior to technical editing, formatting for publication and author proofing. The American Chemical Society provides "Just Accepted" as a free service to the research community to expedite the dissemination of scientific material as soon as possible after acceptance. "Just Accepted" manuscripts appear in full in PDF format accompanied by an HTML abstract. "Just Accepted" manuscripts have been fully peer reviewed, but should not be considered the official version of record. They are accessible to all readers and citable by the Digital Object Identifier (DOI@). "Just Accepted" is an optional service offered to authors. Therefore, the "Just Accepted" Web site may not include all articles that will be published in the journal. After a manuscript is technically edited and formatted, it will be removed from the "Just Accepted" Web site and published as an ASAP article. Note that technical editing may introduce minor changes to the manuscript text and/or graphics which could affect content, and all legal disclaimers and ethical guidelines that apply to the journal pertain. ACS cannot be held responsible for errors or consequences arising from the use of information contained in these "Just Accepted" manuscripts. 


\title{
$30 \mathrm{GHz}$ optoelectronic mixing in CVD
}

\section{graphene}

\author{
Alberto Montanaro, ${ }^{* \dagger}$ Sana Mzali, ${ }^{\dagger}$ Jean-Paul Mazellier, ${ }^{\dagger}$ Odile Bezencenet, ${ }^{\dagger}$ \\ Christian Larat, $^{\dagger}$ Stephanie Molin, ${ }^{\dagger}$ Loïc Morvan, ${ }^{\dagger}$ Pierre Legagneux, $^{\dagger}$ Daniel \\ Dolfi, ${ }^{\dagger}$ Bruno Dlubak, ${ }^{\ddagger}$ Pierre Seneor, ${ }^{\ddagger}$ Marie-Blandine Martin, $₫$ Stephan \\ Hofmann, John Robertson, Alba Centeno, ${ }^{\S}$ and Amaia Zurutuza ${ }^{\S}$ \\ Thales Research and Technology, 1, Avenue Augustin Fresnel, 91767 Palaiseau, France, \\ Unité Mixte de Physique CNRS/Thales, 1, Avenue Augustin Fresnel, 91767 Palaiseau, \\ France, Department of Engineering, University of Cambridge, Cambridge CB21PZ, United \\ Kingdom, and Graphenea S.A., Tolosa Hiribidea, 76 E-20018 Donostia, Spain \\ E-mail: alberto.montanaro@thalesgroup.com
}

\begin{abstract}
The remarkable properties of graphene, such as broadband optical absorption, high carrier mobility and short photogenerated carrier lifetime, are particularly attractive for high-frequency optoelectronic devices operating at $1.55 \mu \mathrm{m}$ telecom wavelength. Moreover, the possibility to transfer graphene on a silicon substrate using a CMOScompatible process opens the ability to integrate electronics and optics on a single
\end{abstract}

\footnotetext{
*To whom correspondence should be addressed

${ }^{\dagger}$ Thales Research and Technology, 1, Avenue Augustin Fresnel, 91767 Palaiseau, France

$\ddagger$ Unité Mixte de Physique CNRS/Thales, 1, Avenue Augustin Fresnel, 91767 Palaiseau, France

IDepartment of Engineering, University of Cambridge, Cambridge CB21PZ, United Kingdom

${ }^{\S}$ Graphenea S.A., Tolosa Hiribidea, 76 E-20018 Donostia, Spain
} 
cost-effective chip. Here, we report an optoelectronic mixer based on chemical vapourdeposited graphene transferred on an oxidized silicon substrate. Our device consists in a coplanar waveguide that integrates a graphene channel, passivated with an atomic layer-deposited $\mathrm{Al}_{2} \mathrm{O}_{3}$ film. With this new structure, $30 \mathrm{GHz}$ optoelectronic mixing in commercially-available graphene is demonstrated for the first time. In particular, using a $30 \mathrm{GHz}$ intensity-modulated optical signal and a $29.9 \mathrm{GHz}$ electrical signal, we show frequency downconversion to $100 \mathrm{MHz}$. These results open promising perspectives in the domain of optoelectronics for radar and radio-communication systems.

Keywords: graphene, optoelectronics, optoelectronic mixer, graphene optoelectronics, graphene devices, signal processing, electronics

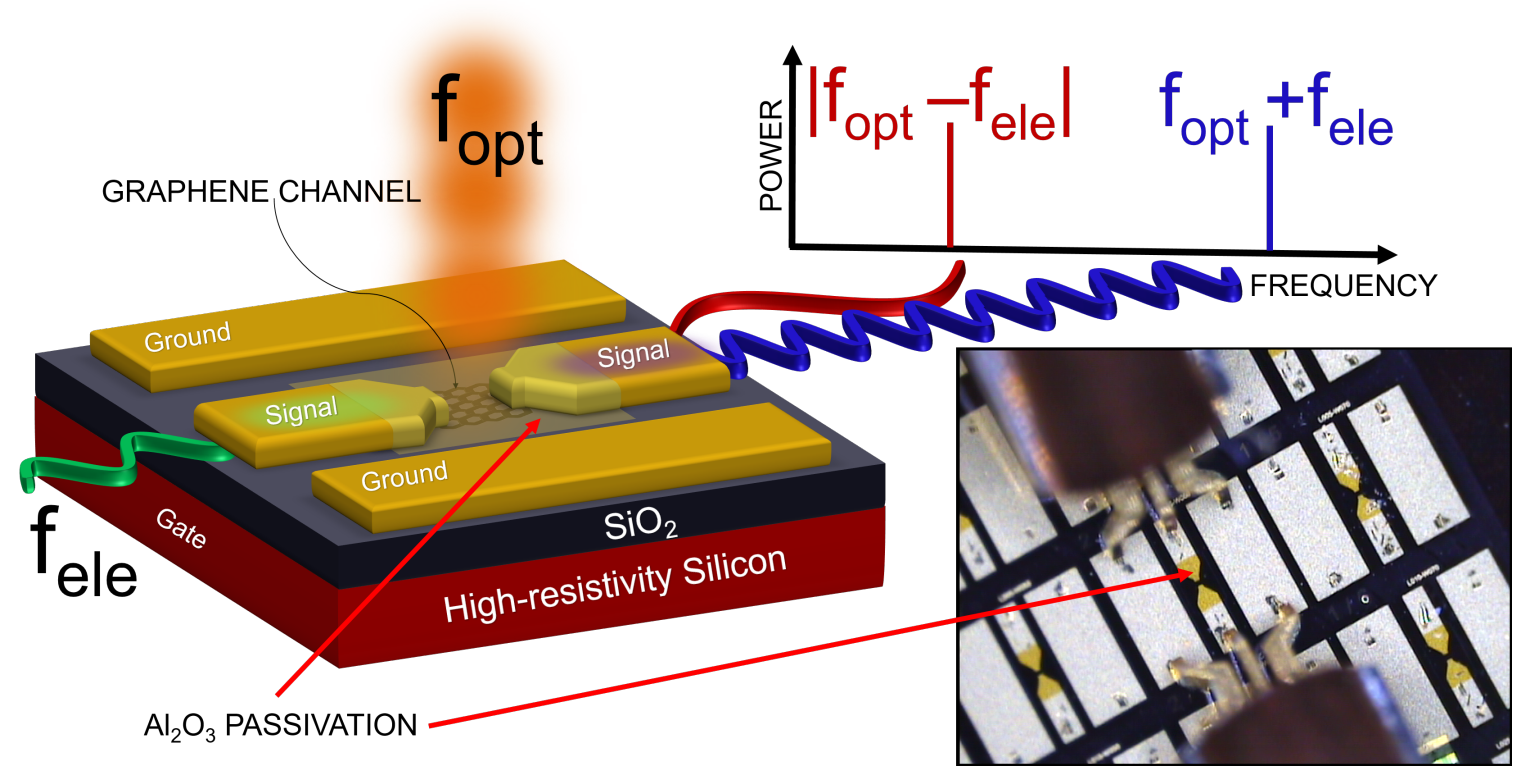

Figure 1: Schematic of our new graphene-based optoelectronic mixer (gOEM). The simultaneous injection of an intensity-modulated laser beam at frequency $f_{\text {opt }}$ and an electrical RF signal at frequency $f_{\text {ele }}$ produces at the output two signals at the difference and sum of the input frequencies. On the bottom right side: Optical image of the gCPW, contacted by the $\mathrm{RF}$ probes. The graphene channel is passivated with an $\mathrm{Al}_{2} \mathrm{O}_{3}$ layer. To allow the RF probes to electrically contact the device, the insulating film has been removed on the metallic pads. 
In high-speed communication systems, information transmission is carried on high-frequency (tens of $\mathrm{GHz}$ ) signals. Once the information is received, a direct processing at the carrier frequency is inconvenient, because it requires high-frequency electronic components. For this reason, a downconversion of the received signal is performed. Standard (low-frequency) electronics can then be used to process the information.

The downconversion function is commonly performed using mixers which demodulate highfrequency carriers to base-band, by multiplying the carrier signal with a Local Oscillator (LO) signal. In many systems, one of the two signals is an optical one. In large RADAR systems, for instance, the LO signal can be advantageously distributed to each antenna using an optical carrier. In this case, the LO signal has to be photodetected to be converted in the electrical domain. Then, the mixing is performed trough conventional electronic mixers. A more compact alternative is based on Optoelectronic Mixers (OEMs), which incorporate in the same device the photodetection and mixing function. More precisely, OEMs are devices that mix an electrical signal at frequency $f_{\text {ele }}$ and an intensity-modulated optical signal at frequency $f_{\text {opt }}$. Two electrical signals are generated at frequencies $f_{\text {up }}=f_{\text {opt }}+f_{\text {ele }}$ (up-conversion) and at $f_{\text {down }}=\left|f_{\text {opt }}-f_{\text {ele }}\right|$ (down-conversion). Performing simultaneously detection and mixing within the same device (self-mixing) reduces the number of components and potentially adds less noise in an optoelectronic system. All this features are particularly attractive for RADAR and LIDAR (Light Detection And Ranging) systems. ${ }^{1-4}$

We focused our study on frequency downconversion, which is needed for the above-mentioned application. However, both downconversion and upconversion are useful functions in an optoelectronic system. For example, in radio-over-fiber systems, the signal is upconverted to millimeter-wave frequency before being radiated by antennas to the end users. ${ }^{5}$

State of the art OEMs that operate at $1.55 \mu \mathrm{m}$ wavelength are based on III-V semiconductors epitaxially grown on InP substrates. $60 \mathrm{GHz}$ optoelectronic mixing has been demonstrated with heterojunction phototransistors (HPT) based on InP-InGaAs, ${ }^{6}$ and downconversion of $0.1 \mathrm{THz}$ signals has been performed using Travelling-Wave Uni-Travelling carrier 
Photodiodes(TW-UTC-PD). ${ }^{7}$ The research for low-cost and CMOS-compatible OEMs is active. $60 \mathrm{GHz}$ bandwidth silicon-based OEMs operating at $0.85 \mu \mathrm{m}$ have been proposed. ${ }^{8}$ However, optical communication systems are mainly based on $1.55 \mu \mathrm{m}$ band, and silicon is not suitable for efficient photodetection at this wavelength. Graphene can overcome the photodetection inefficiency of silicon, because it absorbs a broad spectrum of wavelengths, ${ }^{9}$ including $1.55 \mu \mathrm{m}$. Moreover, graphene exhibits very high carrier mobility and photocarrier short lifetime. Thanks to these remarkable properties, graphene is highly attractive in the domain of high-frequency optoelectronics. Graphene-based photodetectors exhibit an intrinsic bandwidth that may exceed $500 \mathrm{GHz},{ }^{10}$ and CMOS-compatible graphene photodetectors have been demonstrated. ${ }^{11}$ The combination of these characteristics, in junction with the strong efforts done for the realization of cost-effective production techniques for large-scale graphene, ${ }^{12,13}$ makes this material a very good candidate for high-frequency low-cost OEMs, even if the demonstrated sensitivity remains quite limited.

Mao et al. ${ }^{14}$ have fabricated the first graphene OEM based on a field effect transistor. In their work, the graphene channel is biased with a source-drain DC voltage and electrostatically doped by the gate voltage. They have demonstrated the mixing of a $2 \mathrm{MHz}$ electrical signal with a $1 \mathrm{GHz}$ intensity-modulated optical signal at $1.55 \mu \mathrm{m}$ wavelength.

Here we propose a new OEM structure that consists in a CVD graphene-based coplanar waveguide $(\mathrm{gCPW})$. The graphene channel Fermi level was set near the charge neutrality point (CNP), where the electron and hole concentrations are equal. No DC bias is applied to our device when used as an OEM, to minimize power consumption. With this new structure, $30 \mathrm{GHz}$ optoelectronic mixing in commercially-available graphene is demonstrated for the first time. In particular, using a $30 \mathrm{GHz}$ intensity-modulated optical signal and a $29.9 \mathrm{GHz}$ electrical signal, we show frequency downconversion to $100 \mathrm{MHz}$. 


\section{Fabrication and experimental setup}

Figure 1 shows the operation principle of our graphene OEM and (on the bottom right side) a top view optical image of the device, contacted by RF electrical probes. We used a groundsignal-ground configuration, in which the central line (signal line) integrates a $23 \mu \mathrm{m}$ long and $20 \mu m$ wide graphene channel.

To fabricate the device, a CVD graphene monolayer was first transferred on a high-resistivity silicon substrate covered by a $2 \mu m$ thick thermal $\mathrm{SiO}_{2}$ layer. Then, the graphene active zone was defined by optical lithography and reactive ion etching. A metallic multilayer (Ni-Ti-Au$\mathrm{Ti}$ ) was deposited by electron beam evaporation, and an optical lithography pattern allowed us to locally grow $2 \mu \mathrm{m}$ thick pads by electrolytic process, which reduces RF losses. The metallic multilayer deposited on the graphene channel was removed by successive etching techniques (dry/wet) until the Ni base layer was removed by wet chemistry. Finally, a 30 nm thick $\mathrm{Al}_{2} \mathrm{O}_{3}$ layer was deposited by Atomic Layer Deposition (ALD) to passivate the graphene channel. ${ }^{31}$

The comparison between the Raman spectra performed after graphene transfer (i.e, before the lithographic process) and after passivation, indicates that the structural properties of graphene are not degraded by the fabrication process (see Supporting Information).

The average graphene grain size was a few microns. The field effect mobility was around $2000 \frac{\mathrm{cm}^{2}}{V s}$, which is a typical value for such grain size, according to Petrone et al. ${ }^{32}$

Figure 2 shows the experimental setup. We used a $1.55 \mu \mathrm{m}$ laser, intensity-modulated with a Mach-Zehnder Modulator(MZM). An Erbium-doped fiber amplifier (EDFA) amplifies the modulated laser beam power, which is guided and focused on the CPW graphene channel. Two high-frequency probes contact the CPW ports. Both probes are connected to a bias tee to decouple the DC and the modulated components of the electrical signals, and allow DC biasing of each port. High frequency electrical signals are injected in the input port, while the output CPW port transfers the processed electrical signal to a spectrum analyzer. Finally, the high-resistivity silicon substrate is contacted and used as back-gate ( $V_{G}$ in figure 
2), to control the graphene Fermi-level with respect to the CNP.

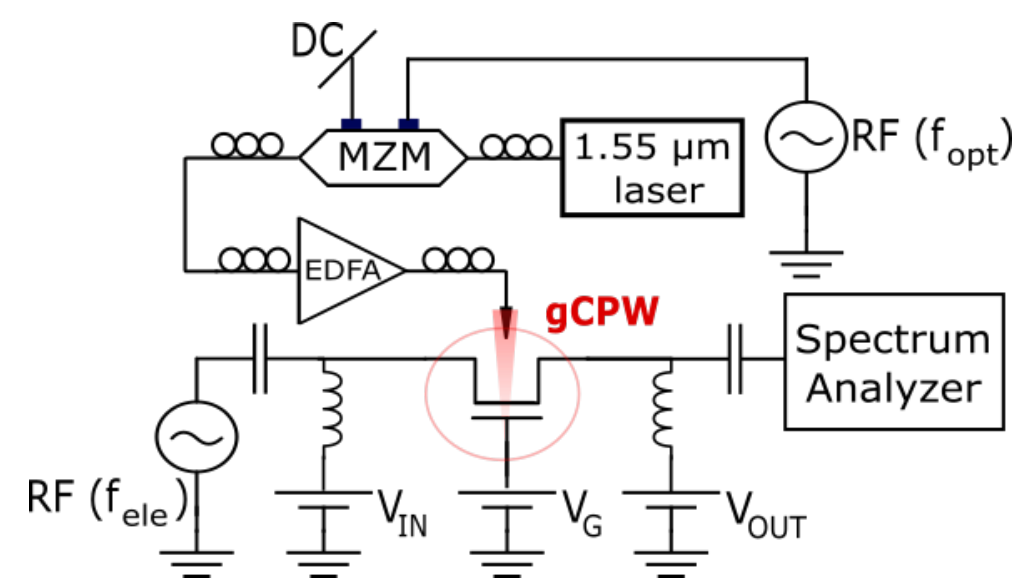

Figure 2: Experimental scheme. $V_{I N}$ : DC Input Voltage; $V_{O U T}$ : DC output voltage; $V_{G}$ : Voltage applied on the high-resistivity silicon substrate, acting as back-gate. MZM: MachZehnder Modulator; EDFA: Erbium-doped fiber amplifier; gCPW: graphene coplanar waveguide

\section{Results}

Figure 3a shows the DC current that flows in the graphene channel of the device, as a function of the back-gate voltage, for a DC channel bias $V_{D C}=V_{I N}-V_{O U T}=4 \mathrm{~V}$ (see blue solid line in figure 3a). Thanks to the $\mathrm{Al}_{2} \mathrm{O}_{3}$ passivation layer, time-stable $\mathrm{V}$-shaped curves are obtained $^{15}$. The measurement allowed us to determine the CNP voltage $\left(V_{C N P}\right)$. Then, the biased channel was illuminated by a laser modulated in intensity at a frequency $f_{\text {opt }}=$ $5 \mathrm{GHz}$. The modulated component of the optical power on the channel was $P_{m}=22.5 \mathrm{~mW}$. In the same figure $(3 \mathrm{a})$ the amplitude of the generated $\mathrm{AC}$ photocurrent $\left(I_{p h, m}\right)$ is plotted as a function of the back-gate voltage (red dots). One can see that the photodetection has its maximal efficiency when the channel current is minimum. This behavior has already been observed by Freitag et al. in lightly doped graphene. ${ }^{16}$ According to theoretical studies, ${ }^{17-19}$ this is due to the decrease of the hot carriers lifetime with increasing carrier density. For this reason, all the following measurements have been performed setting $V_{G}=V_{C N P}$. 
We performed two types of experiments. First, we characterized the device by maintaining a DC channel bias voltage (photodetection). Then, the constant bias was switched off and an RF signal was injected (optoelectronic mixing).

\section{Photodetection}

For the same laser parameters of figure $3 \mathrm{a}$, figure $3 \mathrm{~b}$ shows the amplitude of $I_{p h, m}$ as a function of the channel bias. The dependence is linear for voltages up to $6 \mathrm{~V}$. The $I_{p h, m}$ amplitude value obtained for $V_{D C}=8 \mathrm{~V}$ suggests that the photocurrent response starts to saturate for voltages above $6 \mathrm{~V}$. Such a behavior has already been observed. ${ }^{16}$

Figure 3c shows that the amplitude of $I_{p h, m}$ varies linearly with $P_{m}\left(P_{m}\right.$ varies between 0 and $22.5 \mathrm{~mW}$ ). The modulation frequency is still $f_{\text {opt }}=5 \mathrm{GHz}$ and the channel bias was $V_{D C}$ $=6 \mathrm{~V}$

For the same channel bias, The frequency response of the device is presented in figure $3 \mathrm{~d}$. Here, the photoresponsivity (namely, the photocurrent amplitude $I_{p h, m}$ normalized over the modulated component of the optical power $P_{m}$ ) is plotted as a function of $f_{\text {opt }}$ for frequencies up to $30 \mathrm{GHz}$.

Figure $3 \mathrm{~b}$ and $3 \mathrm{c}$ demonstrate that the photocurrent is a linear function of both the channel bias and the optical power. The photocurrent expression of a photodetector operating in such a linear regime can be written as:

$$
I_{p h}=\alpha P_{o p t} V_{\text {bias }}
$$

where $P_{\text {opt }}$ is the optical incident power, $V_{\text {bias }}$ is the channel bias and $\alpha$ is a proportionality constant $\left[1 / V^{2}\right]$, which sizes the sensitivity of the device. In the experimental configuration described above, $V_{\text {bias }}=V_{D C}$ and $P_{o p t}=P_{c w}+P_{m} \sin \left(2 \pi f_{o p t} t\right)$, where $P_{c w}$ and $P_{m}$ are the amplitudes of, respectively, the constant and modulated components of the intensity- 


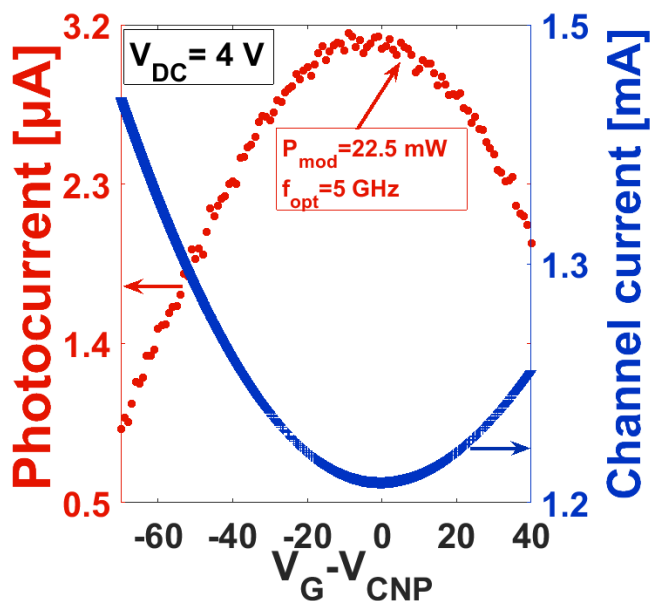

(a)

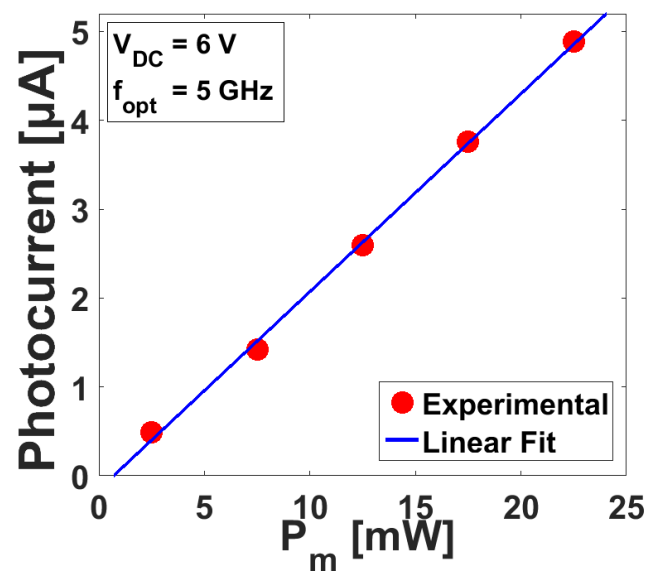

(c)

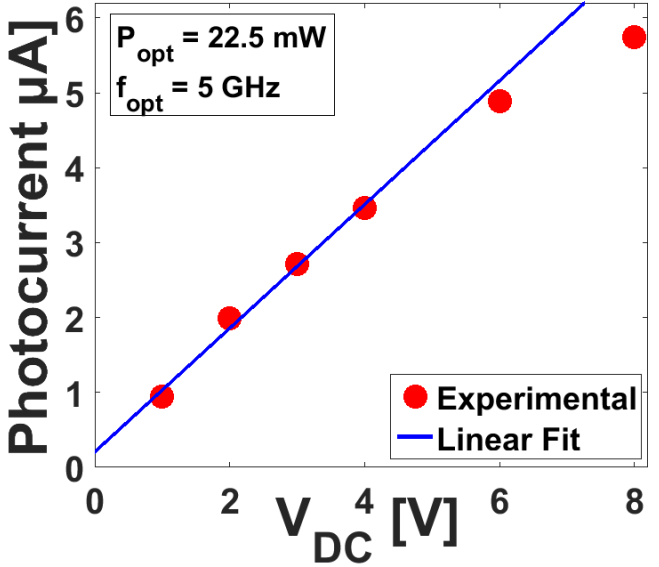

(b)

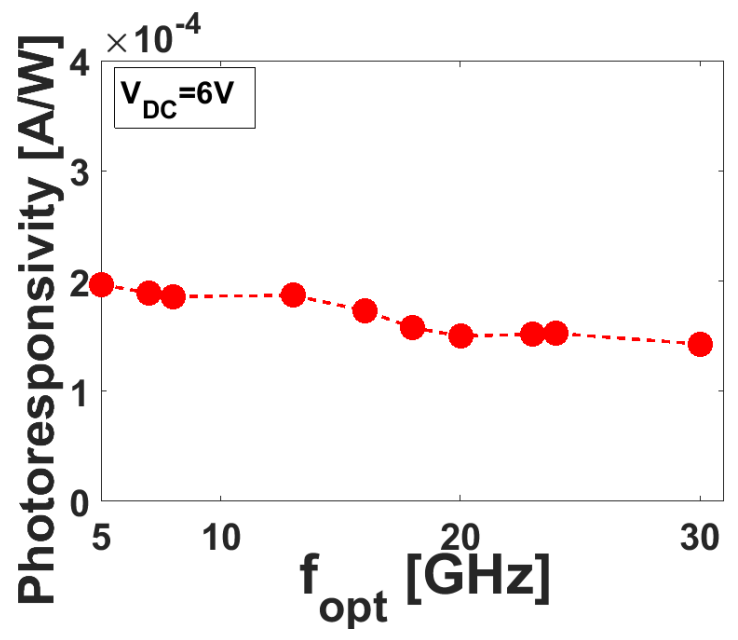

(d)

Figure 3: (a) blue plot: channel current as a function of $V_{G}-V_{C N P}$; red plot: photocurrent generated by a $5 \mathrm{GHz}$ intensity-modulated laser beam. The modulated component of the laser beam is fixed at $P_{m}=22.5 \mathrm{~mW}$. Both curves are measured under a channel bias voltage $V_{D C}=4 V$.(b) Photocurrent as a function $V_{D C}$ for $P_{m}=22.5 \mathrm{~mW}\left(f_{\text {opt }}=5 \mathrm{GHz}\right)$; the small residual current at a polarization of $0 \mathrm{~V}$ predicted by the linear fit can be due to the non-perfect symmetry of the device. ${ }^{20}$ Without laser illumination, the measured current was below $0.2 \mu \mathrm{A}$ (corresponding to the noise level of the spectrum analyzer)(c) Photocurrent as a function of $P_{m}$ for $f_{\text {opt }}=5 \mathrm{GHz}$ and $V_{D C}=6 \mathrm{~V}$.(d) Photoresponsivity $\left(I_{p h, m} / P_{m}\right)$ as a function of the optical modulation frequency $f_{\text {opt }}$.

All the values are corrected with respect to the cables and device losses (see Supporting information). 
modulated laser beam, and $f_{\text {opt }}$ is the modulation frequency. We can thus rewrite equation 1 as:

$$
I_{p h}=\alpha P_{c w} V_{b i a s}+\alpha P_{m} V_{b i a s} \sin \left(2 \pi f_{o p t} t\right)=I_{p h, c w}+I_{p h, m}
$$

where $I_{p h, c w}$ is the photocurrent component generated by the constant optical power $P_{c w}$, while the AC photocurrent (measured in plots 3b and 3c) generated by the modulated optical power is:

$$
I_{p h, m}=\alpha P_{m} V_{D C} \sin \left(2 \pi f_{\text {opt }} t\right)
$$

Equation 3 expresses the $\mathrm{AC}$ photocurrent generated when the device is employed as a photodetector.

\section{Optoelectronic mixing}

In the optoelectronic mixing configuration, we switched off the constant voltage bias $V_{D C}$ and injected an RF modulated signal in the gCPW, while maintaining the illumination of the graphene channel. The blue solid line in figure 4a shows the power measured on the spectrum analyzer without any loss correction (see Supporting information). The injected electrical power was $14 \mathrm{dBm}$, at frequency $f_{\text {ele }}=400 \mathrm{KHz}$. $P_{m}$ was set to $22.5 \mathrm{~mW}$, and $f_{\text {opt }}=5$ GHz. Two peaks appear at $f_{\text {down }}=\left|f_{\text {opt }}-f_{\text {ele }}\right|=4.9996 \mathrm{GHz}$ and $f_{\text {up }}=f_{\text {opt }}+f_{\text {ele }}=5.0004$ $\mathrm{GHz}$, experimentally demonstrating the optoelectronic mixing. One can notice that no signal at $f_{\text {opt }}$ is measured (no constant bias $V_{D C}$ was applied). For a direct comparison with the photodetection mode, in the same figure (4a), the red dashed curve shows the power signal when the modulated component of the electrical signal was switched off, and a constant $V_{\text {bias }}=V_{D C}=3 \mathrm{~V}$ was applied.

For the same optical signal $\left(f_{\text {opt }}=5 \mathrm{GHz}\right)$ and the same electrical frequency $\left(f_{\text {ele }}=400 \mathrm{KHz}\right)$, we measured the downconverted power $P_{I F}$ at intermediate frequency $(\mathrm{IF}=4.9996 \mathrm{GHz})$ as a function of the RF electrical input power $\left(P_{I N}\right)$. Figure $4 \mathrm{~b}$ shows that $P_{I F}$ varies linearly with $P_{I N}$. As $P_{I F}$ and $P_{I N}$ are respectively proportional to $I_{p h}^{2}$ and $V_{R F}^{2}, I_{p h}$ varies linearly 
with $V_{R F}$. This confirms that $I_{p h}$ is proportional to the source-drain bias for either DC (figure $3 \mathrm{~b}$ ) or AC electrical signals (4b).

For the optoelectronic mixing configuration, the channel bias in equation 2 is $V_{\text {bias }}=$ $V_{m} \sin \left(2 \pi f_{\text {ele }} t+\phi\right)$, where $V_{m}$ is the amplitude of the RF electrical signal, and $\phi$ is a phase shift with respect to the optical power modulation. $I_{p h, m}$ can thus be rewritten as:

$$
I_{p h, m}=\alpha\left[P_{m} \sin \left(2 \pi f_{o p t} t\right)\right]\left[V_{m} \sin \left(2 \pi f_{\text {ele }} t+\phi\right)\right]
$$

giving:

$$
I_{p h, m}=\frac{\alpha P_{m} V_{m}}{2}\left[\cos \left(2 \pi\left(f_{\text {opt }}-f_{\text {ele }}\right) t-\phi\right)-\cos \left(2 \pi\left(f_{\text {opt }}+f_{\text {ele }}\right) t+\phi\right)\right]
$$

Due to the mixing between the electrical and optical signals, two signals are generated at frequencies $f_{\text {up }}=f_{\text {opt }}+f_{\text {ele }}$ and $f_{\text {down }}=\left|f_{\text {opt }}-f_{\text {ele }}\right|$. This explains the experimental measurements.

We then studied the optoelectronic mixing with high-frequency (close to $30 \mathrm{GHz}$ ) electrical and optical signal carriers. In particular, for $f_{\text {opt }}=30 \mathrm{GHz}$ and $f_{\text {ele }}=29.9 \mathrm{GHz}$, a downconversion to $f_{\text {down }}=100 \mathrm{MHz}$ is obtained (figure 4c).

The $P_{I F}$ values are small (around $-100 \mathrm{dBm}$ ). To estimate the intrinsic efficiency of the OEM, we plotted in figure $4 \mathrm{~d}{\frac{P_{I F}}{P_{I N}}}^{*}$, which is the ratio $\frac{P_{I F}}{P_{I N}}$ corrected over the losses extracted from the S parameter measurements (See supporting information). We performed downconversion experiments for different electrical frequencies. An optical signal at frequency $f_{\text {opt }}$ $=10 \mathrm{GHz}$ and $P_{m}=22.5 \mathrm{~mW}$ was mixed with electrical signals at frequencies $f_{\text {ele }}$ varying from 6 to $9.99 \mathrm{GHz}$. Thus, the corresponding downconverted or intermediate frequency (IF) varied between $10 \mathrm{MHz}$ and $4 \mathrm{GHz}$. The ratio is constant, as it oscillated (only) in a range of about $2 \mathrm{~dB}$ over the entire frequency window. 


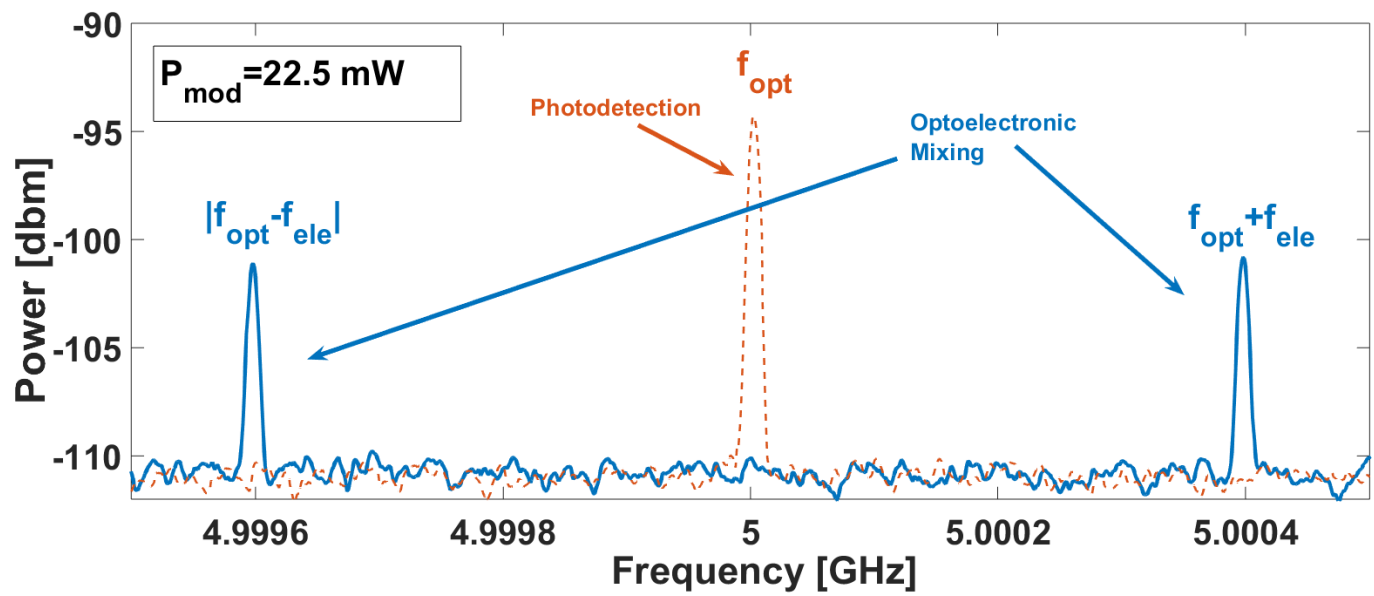

(a)

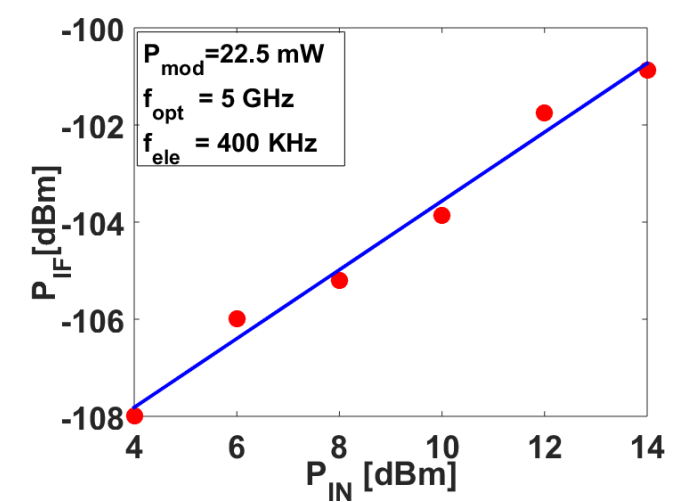

(b)

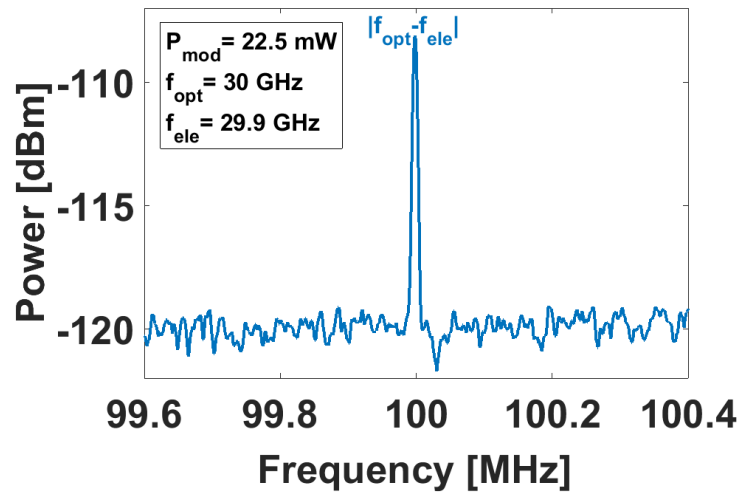

(c)

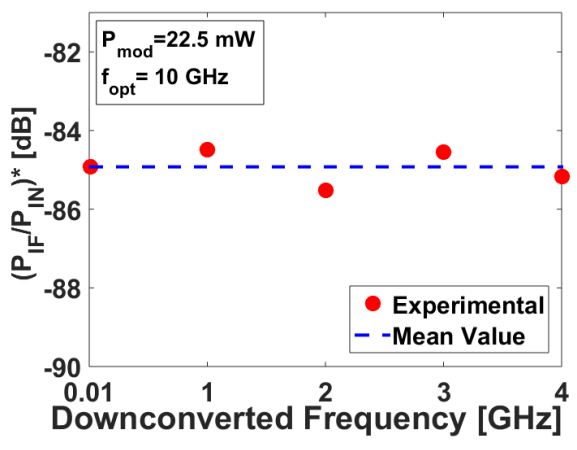

(d)

Figure 4: (a) solid blue curve: optoelectronic mixing effect, obtained by injecting a $14 \mathrm{dBm}$ electrical signal at frequency $f_{\text {ele }}=400 \mathrm{KHz}$. The laser parameters are: $P_{m}=22.5 \mathrm{~mW}$ and $f_{\text {opt }}=5 \mathrm{GHz}$. Red dashed curve (for comparison): photodetection of the modulated component of the laser beam at $5 \mathrm{GHz}$. In this case, $V_{\text {bias }}=V_{D C}=3 \mathrm{~V}$. (b)Downconverted power $\left(P_{I F}\right)$ at intermediate frequency (IF) as a function of the electrical RF input power $\left(P_{I N}\right)$, for the same laser parameters and electrical frequency $(400 \mathrm{KHz})$. (c) Downconverted power to $100 \mathrm{MHz}$ of a laser signal at $f_{\text {opt }}=30 \mathrm{GHz}\left(P_{m}=22.5 \mathrm{~mW}\right)$, mixed with a $10 \mathrm{dBm}$ electrical signal at $29.9 \mathrm{GHz}$. (d) Optoelectronic mixing of a $10 \mathrm{GHz}$ optical signal and an electrical signal at $6,7,8,9,9.99 \mathrm{GHz}$. The plot shows $\frac{P_{I F}}{P_{I N}}$, that is the ratio $\frac{P_{I F}}{P_{I N}}$, from which we subtracted the losses extracted from the $\mathrm{S}$ parameters measurements (see Supporting Information). The laser power is $P_{m}=22.5 \mathrm{~mW}$.

\section{ACS Paragon Plus Environment}




\section{Discussion and Conclusion}

We studied a new high-frequency OEM which relies on a coplanar waveguide (gCPW) integrating a commercially-available CVD graphene channel. A simple model is used to describe its operating behavior This model is based on two linear dependencies, experimentally verified: the photocurrent as a function of the optical incident power $\left(P_{\text {opt }}\right)$ at a fixed voltage drop along the channel, and the photocurrent as a function of the voltage drop $\left(V_{\text {bias }}\right)$ along the channel at a fixed optical power. As the photocurrent is proportional to $P_{\text {opt }} \cdot V_{\text {bias }}$, upconverted and downconverted signals can be generated

Several physical processes can be involved in the generation of a photocurrent in graphene. ${ }^{21}$ In this work, the photocurrent is generated in biased graphene at low electrostatic doping. According to Freitag et al., ${ }^{16}$ the photovoltaic effect, that is the separation of photo-generated electron-hole pairs by an electric field, dominates in this regime. It is important to note that a bolometric effect can induce a change in the number of carriers contributing to the current and that this mechanism coincides with the photovoltaic effect for biased graphene. ${ }^{13}$

To separate electron-hole pairs, a first solution is to exploit the electrical field that builds up at the metal-graphene interface, due to their work-function difference. ${ }^{22}$ Another choice is to generate a uniform electric field along the channel. ${ }^{16}$ The first solution, which employs the constant built-in metal-graphene electric field, is well adapted for photodetection as it avoids biasing the graphene channel, that leads to large parasitic dark current. On the contrary, a modulated electric field is essential for optoelectronic mixing. It can be easily obtained by modulating the channel bias. For this reason, we adopted the second solution. This choice generates DC power consumption when the device is employed as a photodetector, since a DC voltage is applied on the channel. But, when the device is used as an OEM, no DC bias is needed, and no associated power consumption is thus generated.

Our device employs a back-gate just to set the graphene Fermi level near the Dirac point (where the photocurrent in the low-doped regime is maximal). Many efforts in graphene technology concern the fabrication of devices integrating low-doped graphene. With such 
technology, the use of back-gated devices is no longer necessary if their working point is stable and near the CNP.

Used as a photodetector, our device exhibits an intrinsic (i.e. after loss correction as explained in Supporting Information) low photoresponsivity that varies between 142 and 197 $\mu A / \mathrm{W}$ over a wide range of frequencies up to $30 \mathrm{GHz}$. Used as an OEM, we showed optoelectronic mixing of electrical/optical frequencies up to $30 \mathrm{GHz}$. We also showed a flat downconversion response (2 $\mathrm{dB}$ oscillation range) over a large frequency range.

Figure 4 a shows the output power of the device, at a fixed optical input power, for the same electrical power absorbed in the graphene channel in both photodetection and OEM configuration(see Supporting Information). For photodetection, the absorbed electrical power is due to the DC bias, while in the OEM configuration it is due to the RF bias voltage. The output power is $-94 \mathrm{dBm}$ (photodetection) and $-98 \mathrm{dBm}(\mathrm{OEM})$. Since an OEM mixes a photodetected signal with an electrical AC signal, one can conclude that the low output power is mainly due to the small photodetected signal and not to the mixing process itself. The photodetection losses can be attributed to the low photoconductive gain and the weak absorption. The photoconductive gain ${ }^{13}$ is defined as $G=\frac{\tau_{r}}{\tau_{\text {trans }}}$, where $\tau_{r}$ is the recombination time, while $\tau_{\text {trans }}=\frac{L^{2}}{\mu V_{\text {bias }}}$ is the the transit time of a carrier under an electric field generated in the channel. Time-resolved experiments show a first sub picosecond relaxation time due to interactions with optical phonons followed by a picosecond decay ${ }^{23-28}$. By considering a mobility of $2000 \frac{\mathrm{cm}^{2}}{V s}$, that is a typical value obtained in our CVD graphene, and by using a value of $\tau_{r}=1 \mathrm{ps}$, we found for our device a photoconductive gain around $10^{-3}$. This corresponds to a power loss, with respect to the case $\mathrm{G}=1$, around $60 \mathrm{~dB}$.

Moreover, one has to consider the intrinsic limitation of graphene due to a small light absorption (which is however very impressive if we consider that we are dealing with a monoatomic material). The simulation described in the Supporting Information predicts a light absorption of about $1.8 \%$. This corresponds to a power loss of $35 \mathrm{~dB}$ with respect to the ideal case of $100 \%$ absorption. 
To improve the downconversion efficiency without decreasing the operating frequency, it is necessary to increase the light-graphene interaction by using e.g. graphene on silicon photonic waveguides ${ }^{29}$ or antenna-enhanced graphene devices, ${ }^{30}$ and to decrease the transit time $\tau_{\text {trans }}$ using shorter channels.

Our results open interesting perspectives in the utilization of graphene-based devices for the mixing of high-frequency electronic and optical signals in the telecom range with a low-cost CMOS-compatible structure.

\section{Acknowledgement}

This research was supported by the EU FP7 work programme under Grant GRAFOL (No. 285275) and Graphene Flagship (No. 604391). 


\section{Supporting Information Available}

Optical power and photocurrent calculation.

Downconversion efficiency.

Raman spectrum measurements.

Absorbed optical power.

This material is available free of charge via the Internet at http://pubs.acs.org/. 
Note: The authors declare no competing financial interest.

\section{References}

(1) Pillet, G.; Morvan, L.; Dolfi, D.; Huignard, J.-P. Proc. SPIE, 2008, 7114, 71140E71140E-12.

(2) Ruff W. C.; Bruno J. D.; Kennerly S. W.; Ritter K.; Shen P. H.; Stann B. L.; Stead M. R.; Sztankay Z. G.; Tobin M. S. Proc. SPIE, 2000, 4035, 152-162.

(3) Ghelfi P.; Laghezza F.; Scotti F.; Serafino G.; Capria A.; Pinna S.; Onori D.; Porzi C.; Scaffardi M.; Malacarne A. et al. Nature, 2014, 507, 341-345.

(4) Vercesi V.; Onori D.; Laghezza F.; Scotti F.; Bogoni A.; Scaffardi M. Opt. Lett., 2015, 40, 1358-1361.

(5) Harun H.; Idrus S. M.; Mohammad A. B. Research and Development, 200\%. SCOReD 2007. 5th Student Conference on, 2007, p. 1-5.

(6) Choi C.-S.; Seo J.-H.; Choi W.-Y.; Kamitsuna H.; Ida M.; Kurishima K. Photonics Technology Letters, IEEE, 2005, 17, 2721-2723.

(7) Rouvalis E.; Fice M. J.; Renaud C. C.; Seeds A. J. Opt. Express, 2011, 19, 2079-2084.

(8) Kang H.-S.; Choi W.-Y. Microwave Symposium, 200\%. IEEE/MTT-S International, 2007, p. 233-236.

(9) Nair R. R.; Blake P.; Grigorenko A. N.; Novoselov K. S.; Booth T. J.; Stauber T.; Peres N. M. R.; Geim A. K. Science, 2008, 320, 1308.

(10) Xia F.; Mueller T.; Lin Y.-m.; Valdes-Garcia A.; Avouris P. Nat Nano, 2009, 4, 839843. 
(11) Pospischil A.; Humer M.; Furchi M. M.; Bachmann D.; Guider R.; Fromherz T.; Mueller T. Nat Photon, 2013, 7, 892-896.

(12) Kidambi P. R.; Ducati C.; Dlubak B.; Gardiner D.; Weatherup R. S.; M.-B. Martin; Seneor P.; Coles H.; Hofmann S. The Journal of Physical Chemistry C, 2012, 116, $22492-22501$.

(13) Ferrari A. C.; Bonaccorso F.; Fal'ko V.; Novoselov K. S.; Roche S.; Boggild P.; Borini S.; Koppens F. H. L.; Palermo V.; Pugno N. et al. Nanoscale, 2015, 7, 4598-4810.

(14) Mao X.; Cheng C.; Huang B.; Zhang Z.; Gan S.; Chen H.; Chen H. Electron Device Letters, IEEE, 2015, 36, 253-255.

(15) Sagade A. A.; Neumaier D.; Schall D.; Otto M.; Pesquera A.; Centeno A.; Elorza A. Z.; Kurz H. Nanoscale, 2015, 7, 3558-3564.

(16) Freitag M.; Low T.; Xia F.; Avouris P. Nat Photon, 2013, 7, 53-59.

(17) Calandra M.; Mauri F. Phys. Chem. Lett, 2007, 76, 205411.

(18) Tse W.-K.; Das Sarma S. Phys. Rev. Lett., 2007, 99, 236802.

(19) Tse W.-K.; Hwang E. H.; Das Sarma S. Applied Physics Letters, 2008, 93, 023128.

(20) Mueller T.; Xia F.; Avouris P. Nat Photon, 2010, 4, 297-301.

(21) Koppens F. H. L.; Mueller T.; Avouris P.; Ferrari A. C.; Vitiello M. S.; Polini M. Nat Nano, 2014, 9, 780-793.

(22) Lee E.J. H.; Balasubramanian K.; Weitz R. T.; Burghard M.; Kern K. Nat Nano, 2008, 3, 486-490.

(23) Lin K.-C.; Li M.-Y.; Ling D. C.; Chi C. C.; Chen J.-C. Phys. Rev. B, 2015, 91, 125440. 
(24) Gierz I.; Petersen J. C.; Mitrano M.; Cacho C.; Turcu I. C. E.; Springate E.; Stöhr A.; Köhler A.; Starke; U.; Cavalleri A. Nat Mater, 2013, 12, 1119-1124.

(25) Brida D.; Tomadin A.; Manzoni C.; Kim Y. J.; Lombardo A.; Milana S.; Nair R. R.; Novoselov K. S.; Ferrari A. C.; Cerullo G.; Polini M. Nat Commun, 2013, 4, 1987.

(26) Strait J. H.; Wang H.; Shivaraman S.; Shields V.; Spencer M.; Rana F. Nano Lett., 2011, 11, 4902-4906.

(27) Bistritzer R.; MacDonald A. H. Phys. Rev. Lett., 2009, 102, 206410.

(28) George P. A.; Strait J.; Dawlaty J.; Shivaraman S.; Chandrashekhar M.; Rana F.; Spencer M. G. Nano Lett., 2008, 8, 4248-4251.

(29) Schall D.; Neumaier D.; Mohsin M.; Chmielak B.; Bolten J.; Porschatis C.; Prinzen A.; Matheisen C.; Kuebart W.; Junginger B.; Templ W.; Giesecke A. L.; Kurz H. ACS Photonics, 2014, 1, 781-784.

(30) Yao Y.; Shankar R.; Rauter P.; Song Y.; Kong J.; Loncar M.; Capasso F. Nano Lett., 2014, 14, 3749-3754.

(31) Martin M.-B.; Dlubak B.; Weatherup R. S.; Yang H.; Deranlot C.; Bouzehouane K.; Petroff F.; Anane A.; Hofmann S.; Robertson J. et al. ACS Nano, 2014, 8, 7890-7895.

(32) Petrone N.; Dean C. R.; Meric I.; van der Zande A. M.; Huang P. Y.; Wang L.; Muller D.; Shepard K. L.; Hone J. Nano Letters, 2012, 12, 2751-2756. 


\section{Graphical TOC Entry}

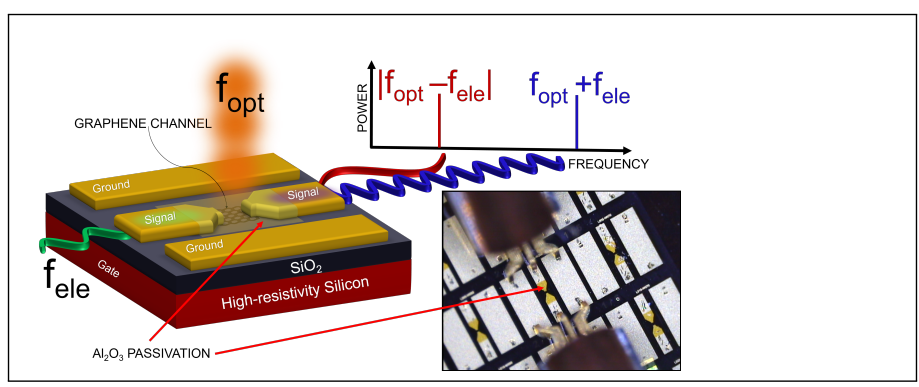

16

17

18

19

20

21

22

23

24

25

26

27

28

29

30

31

32

33

34

35

36

37

38

39

40

41

42

43

44

45

46

47

48

49

50

51

52

53

54

55

56

57

58

59

60 